\title{
Perforación duodenal por barotrauma durante colangiopancreatografía retrógrada endoscópica. Caso clínico
}

\author{
Camilo Heriberto Viñoles-Olid ${ }^{1}$, Jorge Cardozo-Urtaran ${ }^{1}$ y Luis Ruso-Martínez ${ }^{1}$
}

1 Sanatorio Maternidad CASMU IAMPP. Montevideo Uruguay.

Recibido 2020-10-28 y aceptado 2020-11-24

Correspondencia a: Dr. Camilo Heriberto Viñoles-

\section{Duodenal perforation due to barotrauma during endoscopic retrograde cholangiopancreatography. Clinical case}

Introduction: Duodenal perforation during endoscopic retrograde cholangiopancreatography (ERCP) is a rare complication and even more if the mechanism of injury is secondary to barotrauma. The injection of high-pressure-air produces a pneumo-retroperitoneum, the extent and lesional evolution of which is uncertain. Clinical Case: We report the case of a young man who suffered a duodenal perforation during an ERCP, his surgical management and evolution. Discussion: The clinical-imaging diagnosis is usually early and clear if the lesion is detected during the procedure. Its conservative or surgical management will depends on several factors that are analyzed in the present study.

Key words: duodenal perforation; endoscopic retrograde cholangiopancreatography; barotrauma.

\section{Resumen}

Introducción: La perforación duodenal secundaria a la colangiopancreatografía retrógrada endoscópica (CPRE) es una complicación infrecuente y más aún cuando su mecanismo lesional es por barotrauma. La inyección de aire a alta presión produce un neumoretroperitoneo, cuya extensión y evolución lesional es incierta. Caso Clínico: Se comunica el caso de un hombre joven que sufrió una perforación duodenal durante una CPRE, su manejo quirúrgico y evolución. Discusión: El diagnóstico clínico-imagenológico suele ser precoz y claro si se detecta la lesión durante el procedimiento. El manejo terapéutico conservador o quirúrgico de esta entidad depende de varios factores que se analizan en el presente estudio.

Palabras clave: perforación duodenal; colangiopancreatografía retrógrada endoscópica; barotrauma.

\section{Introducción}

La CPRE es un procedimiento que se indica con frecuencia para el tratamiento de enfermedades hepato-bilio-pancreáticas. Sus complicaciones: pancreatitis, sangrado, colangitis y perforación duodenal tienen una incidencia del $5 \%$ al $23 \%$, su mortalidad oscila entre $0,3 \%$ a $1 \% \%^{1,2}$.

La perforación duodenal es la complicación menos frecuente de este procedimiento $(<0,1 \%)$, pero su mortalidad puede ser significativamente mayor (8\%-23\%) como resultado de la sepsis y falla multiorgánica, si existe retraso diagnóstico ${ }^{3}$.

El denominado síndrome de fuga de aire ${ }^{4}$, cuya expresión clínica puede ser neumoperitoneo, neumoretroperitoneo, neumotórax, neumomediastino y enfisema subcutáneo, dependiendo del grado de dispersión del aire, es excepcional, siéndolo más aún su mecanismo de producción vinculado al barotrau$\mathrm{ma}^{3-6} \mathrm{y}$ obedece la inyección de aire sostenida y/o a alta presión en el retroperitoneo, cuya extensión y evolución lesional es incierta, tanto desde el punto de vista mecánico como infeccioso.

Se presenta un caso clínico cuyo interés central es el mecanismo de producción lesional que se postula, realizando análisis de los aspectos diagnósticos, terapéuticos y evolutivos, de esta excepcional situación en el contexto de la literatura sobre el tema. 


\section{Caso Clínico}

Hombre de 45 años, portador de litiasis vesicular, ingresa por cólico biliar, sin ictericia, sin fiebre y tránsito digestivo normal. Al examen: buen estado nutricional, ausencia de signología física abdominal. Paraclínica humoral: hepatograma, amilasemia, hemograma, crasis sanguínea y función renal normales.

Ecografía abdominal muestra una dilatación de la vía biliar intrahepática, vesícula biliar de paredes engrosadas con microlitiasis y una litiasis coledociana.

Se solicita CPRE que informa: papila pequeña, precorte a la hora 11 , Se ingresa varias veces al Wirsung. No es posible ingresar a la vía biliar principal (VBP) en forma segura a través del corte. Queda drenando bilis.

Al finalizar el procedimiento el paciente instala taquicardia, polipnea, dolor epigástrico intenso, con enfisema subcutáneo de cara, cuello, tórax y abdomen, clínico e imagenológico (Figuras 1, 2, 3 ), distensión abdominal difusa y fuga de contraste intestinal, por probable perforación duodenal.

Se decide emplazar drenaje pleural bilateral y laparotomía urgente. Sin peritonitis ni líquido libre en la cavidad abdominal. Maniobra de Kocher. Pequeña área biliosa en cara posterior de duodeno II. Se realiza la colecistectomía y colangiografía transcística a través de la cual se instila azul de metileno que permite evidenciar desgarro duodenal $(1 \mathrm{~cm})$

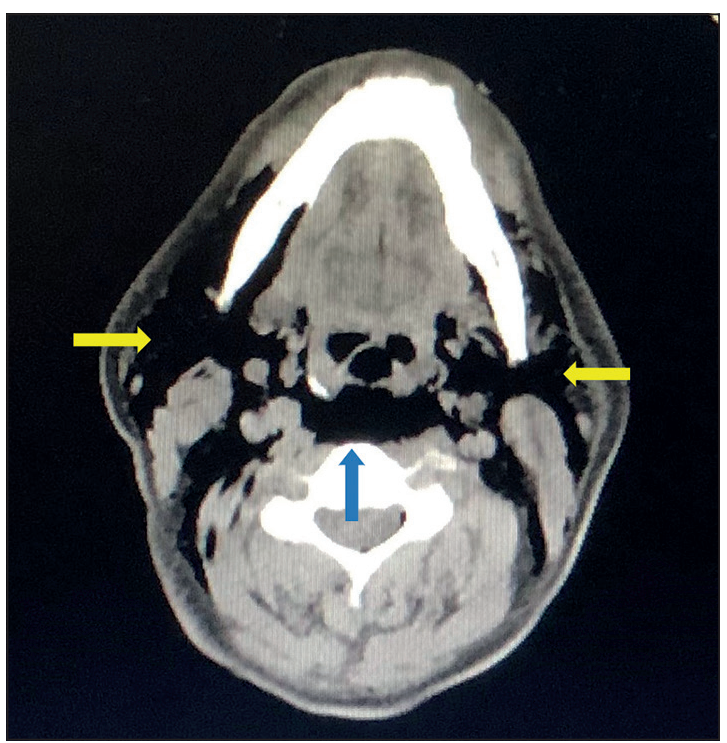

Figura 2. Corte axial cervical de TC, que muestra enfisema de los espacios celulosos de cuello bilateral (flechas amarillas) y prevertebral (flecha azul)

en cara posterior de duodeno II, yuxtapapilar a la ampolla de Vater (Figura 4), la que se sutura en dos planos con hilo reabsorbible, calibrada sobre SNG. Se realiza prueba de hermeticidad, seguido de epiploplastía y drenaje enfrentado a la sutura.

Evolución funcional tórpida, con vómitos reiterados, fiebre y leucocitosis, descenso de la hemoglobina. Una tomografía de control detecta un hematoma del lecho vesicular y el páncreas aumentado de tamaño.

Buena evolución ulterior, con tratamiento médico. Colangiografía transcística de control normal. Se retira el drenaje transcístico. Alta a domicilio a los 27 días de posoperatorio.

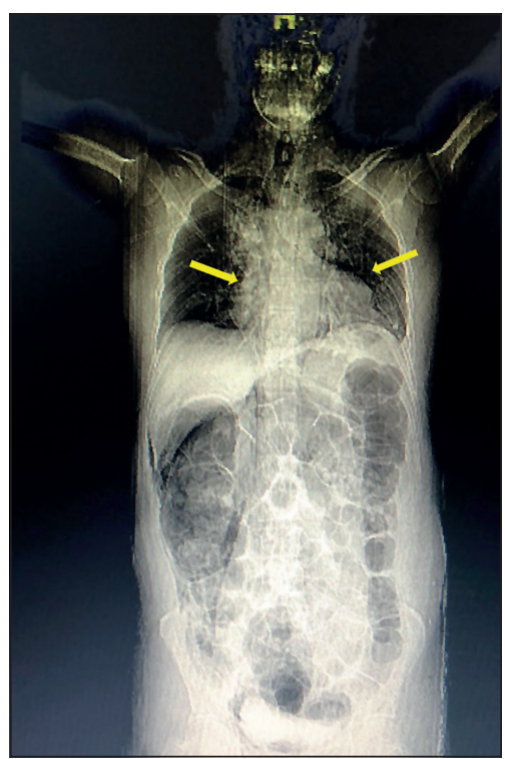

Figura 1. Tomografía toraco-abdominal. Scout-view que permite observar neumotórax bilateral y neumomediastino. Las flechas (amarillas) muestran ambos pulmones retraídos. En abdomen se observa la distensión aérea difusa de todo el tracto digestivo.

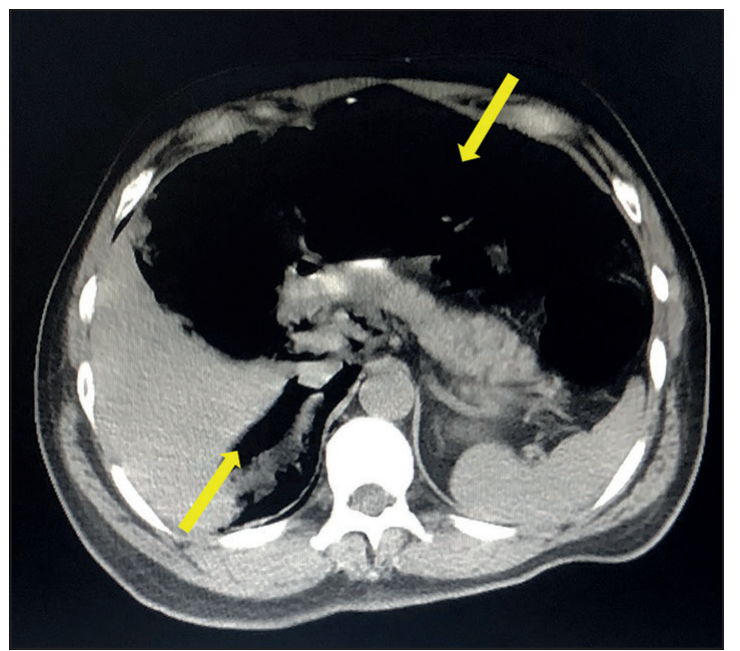

Figura 3. Corte axial de TC de abdomen que evidencia neumoperitoneo extenso (flecha azul) y neumoretroperitoneo (flecha amarilla). 


\section{CASOS CLÍNICOS}

Figura 4. Cirugía. Perforación en cara posterior de segunda porción duodenal. de TC de abdomen que evidencia colección subhepática de $126 \mathrm{~mm}$ de diámetro máximo.
Figura 5. Corte axial
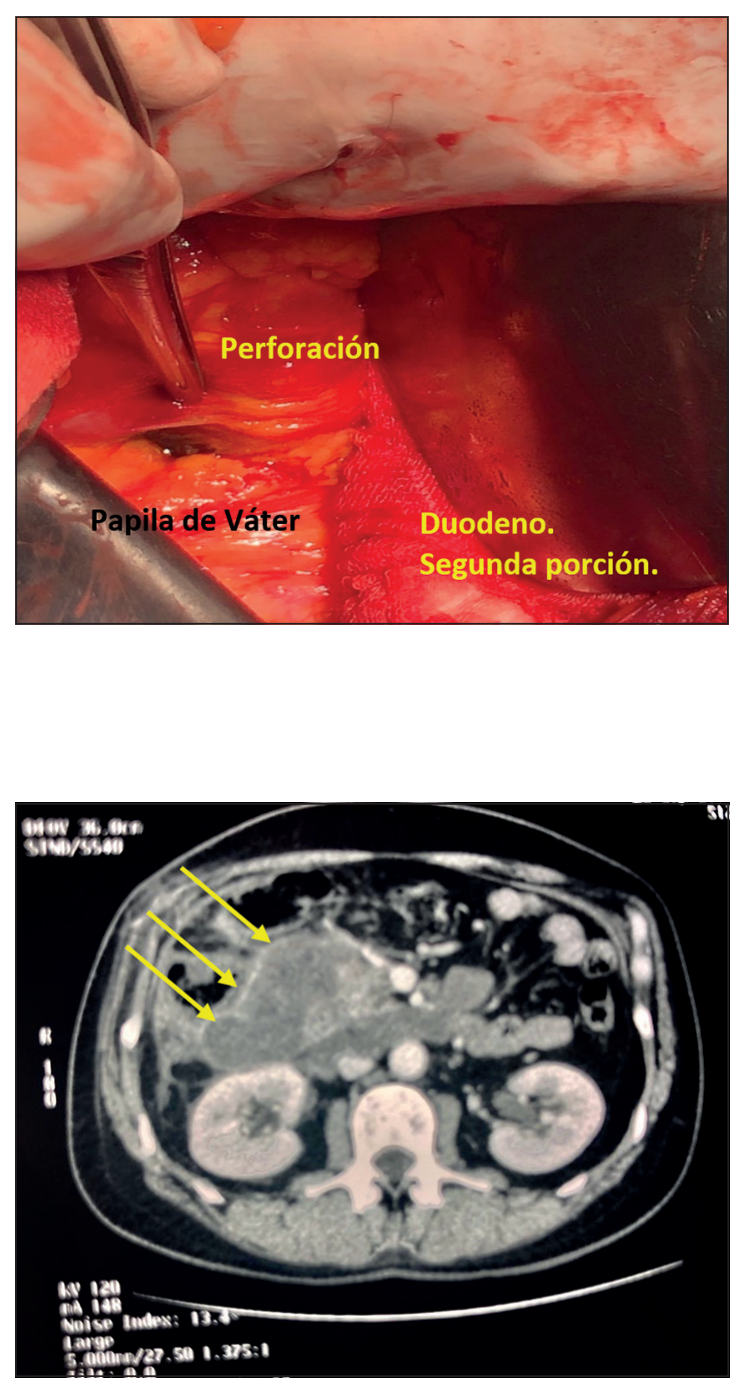

Figura 6. Corte axial de TC de abdomen que evidencia disminución significativa del tamaño de las colecciones. Tejido de densidad de partes blandas por detrás del páncreas.
Reingresa a los 35 días de posoperatorio por dolor abdominal y fiebre. Se realiza tomografía que se observa en la Figura 5, que muestra una colección subhepática.

Se realiza punción percutánea que informa hematoma subhepático. No se obtiene líquido, aun así, se deja un drenaje. Antibioticoterapia con ampicilina sulbactam y metronidazol. Cultivo desarrolla Klebsiella pneumoniae. Rotación de antibiótico a trimetroprim-sulfametoxazol y continúa con lavados por el drenaje. Se mantiene con antibioticoterapia prolongada por 45 días con buena evolución ulterior y alta hospitalaria.

A los 45 días del egreso se realiza tomografía (Figura 6) que muestra pequeña colección residual y la epiploplastía retropancreática. Paciente asintomático a 8 meses de seguimiento.

Esta comunicación cumple las normas Éticas de la Declaración de Helsinki (última modificación octubre de 2013), del Decreto No. 379/008 (Ley de Protección de datos personales/Uruguay).

\section{Discusión}

El diagnóstico inmediato de una perforación duodenal, puede realizarse durante el estudio de CPRE y se define como la presencia de gas o contenido intestinal fuera del tracto digestivo ${ }^{7}$.

En cerca de la mitad de los casos (41\%) el mecanismo de perforación durante CPRE se vincula a una esfinterotomía, inserción o manipulación del endoscopio $(26 \%)$, lesión por guía $(15 \%)$, dilatación de estenosis (3\%), colocación o migración de stent en $2 \%$ de los casos y desconocido en el $7 \% \%^{8}$. Asimismo, se han identificado factores de riesgo para esta complicación dependientes del paciente: disfunción del esfínter de Oddi, sexo femenino, edad avanzada, niveles normales de bilirrubina, distorsión anatómica tipo Billroth II y dependientes del procedimiento: esfinterotomía (precorte) más allá de la hora 11 , inyección de contraste en el Wirsung, dilatación con balón y endoscopista poco experimentado ${ }^{9-11}$. Así, como en este caso, una canulación biliar dificultosa y precorte pueden condicionar edema local que ulteriormente impida la visualización de la lesión provocada.

Las explicaciones que se postulan para la presencia simultánea de neumoperitoneo, neumomediastino y enfisema subcutáneo son: la perforación mucosa que permite que el aire insuflado entre en la pared y diseccione el espacio submucoso y en su progresión desgarre la misma ${ }^{4}$, además, la insuflación de aire presurizado para mantener la permeabi- 
lidad de la luz del tracto digestivo en combinación con una pequeña perforación iatrogénica no detectada durante el procedimiento, puede extenderse ampliamente a través del retroperitoneo ${ }^{6}$. Esto puede haber ocurrido en nuestro caso, donde es evidente que la alta presión sostenida provocó una distensión difusa de todo el tracto digestivo (Figura 1) y una lesión desgarrante -por disociación de planos de adentro/afuera a través de la pared duodenal-y cuya topografía yuxtapapilar y el edema local, facilitaron que pasara desapercibida durante el estudio; aunque no impidió visualizar la fuga de contraste durante la tomografía.

Se ha descrito una probable ruta anatómica por la cual el aire se difunde. Los espacios celulosos del cuello, tórax y abdomen contienen cuatro regiones definidas: tejido subcutáneo, tejido prevertebral, espacio visceral y espacio previsceral. El espacio visceral contiene la tráquea y el esófago y acompaña a estas estructuras en el mediastino y a las vainas broncovasculares. En el sector bajo hay continuidad a través del hiato diafragmático con el espacio de tejido laxo retroperitoneal y peritoneal. Así, hay continuidad a lo largo del cuello, tórax y abdomen. El aire que surge en cualquiera de estas regiones podría llegar a otra área transcurriendo a lo largo de los planos fasciales ${ }^{2-4}$.

Otra explicación la ha dado Kirschner-citado por Schepers ${ }^{2}-$, quien atribuye la diseminación del aire al paso transfrénico de fluidos o gases a través de los poros congénitos o adquiridos en los llamados "síndromes de diafragma poroso".

Nuestro paciente presentaba una lesión de tipo I de Stapfer ${ }^{12}$ (Tabla 1), yuxtapapilar, de tipo desgarro, probable consecuencia de un defecto de desinserción por barotrauma, cuya entidad queda de manifiesto por la producción de un extenso enfisema retroperitoneal, neumotórax bilateral, neumoperitoneo. La entidad de estos hallazgos no se correlaciona con el tamaño de la perforación, sino con la presión y el volumen de aire insuflado a través de la misma.

La existencia en este caso de un neumotórax bilateral estimula considerar su origen en un mecanismo de alta presión y de gravedad potencial. Su diagnóstico es indicación de inmediato drenaje, ya que existen comunicaciones de neumotórax hipertensivo post-CPRE 5 .

Schapers ${ }^{2}$, publica la única revisión de la literatura sobre el tema, con 20 casos, centrado en la producción de neumotórax, aunque la gran mayoría de los casos (18 en 20) la distribución del aire es amplia y variable (neumomediastino, neumotórax, neumoretroperitoneo y enfisema subcutáneo). Ocho casos ocupan los 4 compartimientos, 6 casos 3 de ellos, 4 casos ocupan dos. El tratamiento fue conservador y la serie muestra una mortalidad de un caso. Menciona como factores de riesgo de perforación la existencia de un divertículo yuxtapapilar y la esfinterotomía. Vincula la patogenia con el rápido pasaje de aire al retroperitoneo, de cuyo volumen depende su grado de expansión, pero no menciona específicamente el mecanismo de barotrauma ${ }^{2}$.

Posteriormente a ese estudio localizamos solo tres casos clínicos ${ }^{3,4,6}$. Del análisis de los mismos surge que la mayoría fueron pacientes añosos, afectados de litiasis coledociana, con factores de riesgo de dificultades durante la CPRE: divertículo duodenal, Billroth II previo y el recambio de un stent biliar. En todos los pacientes la distribución del aire fue extensa, aunque en dos de los casos no se identificó la lesión y dos evolucionaron bien con tratamiento conservador. Un caso $^{3}$, con lesión amplia y tratamiento quirúrgico, falleció. Estos $\operatorname{casos}^{3,4,6}$ y Doerr ${ }^{5}$, explican la amplia disección de los espacios celulosos por un mecanismo de barotrauma.

La tomografía con contraste vía oral es el estudio indicado y debe realizarse de forma inmediata si se sospecha una perforación duodenal, buscando identificar aire fuera del tubo digestivo o extravasación de contraste (Figuras 1, 2, 3, 4). La presencia de aire en el retroperitoneo en la tomografía puede verse hasta en un $29 \%$ de los pacientes post-CPRE sin consecuencias patológicas, y los pacientes asintomáticos no tienen indicación quirúrgica.

El endoscopista debe revisar cuidadosamente la papila y la pared del duodeno durante el proce-

\section{Tabla 1. Clasificación de Stapfer ${ }^{12}$. Utilizada para las perforaciones duodenales poscolangiografía, basada en el mecanismo lesional y la localización anatómica}

Tipo I: Perforación duodenal de la pared lateral o medial causada por duodenoscopio.

Habitualmente grande y lejos de la ampolla.

Tipo II: Perforación periampular causada por la esfinterotomía.

Generalmente es pequeña y con mínima fuga de contraste. El diagnóstico puede confirmarse con TC y requiere cirugía con menor frecuencia.

Es la más frecuente y se da en $50 \%$ de los casos.

Tipo III: Perforación del conducto biliar causada por guía o instrumentación con cestilla de Dormia.

Lesiones pequeñas que no producen colecciones y pueden tratarse de forma conservadora.

Tipo IV: Presencia de aire retroperitoneal. No se considera una verdadera perforación y es de manejo conservador. 
dimiento y estar atento a la presencia de aire fuera del tubo digestivo en la radioscopía, así como de la producción de enfisema subcutáneo como presentó este paciente. La infusión de medio de contraste a través del endoscopio es útil para pesquisar fugas. La endoscopía tiene relativa eficacia para detectar perforaciones, más aun considerando que usualmente no se observa la lesión durante el procedimiento endoscópico ${ }^{2}$.

Luego de realizado el procedimiento, el diagnóstico debe sospecharse si el paciente presenta dolor abdominal intenso, lumbalgia o reacción peritoneal. La taquicardia y la fiebre aparecen más tardíamente. El retardo diagnóstico, luego de las 24 horas posteriores, agrava el pronóstico de estos pacientes y aumenta la mortalidad ${ }^{1,7,8,9,11,13}$, debido a la inflamación y el edema local que hace más difícil la movilización del duodenopáncreas para una correcta exposición del duodeno

El objetivo fundamental en estos pacientes es evitar la secuencia infección, sepsis y muerte ${ }^{14}$.

En este sentido, la existencia de un extenso neumoretroperitoneo, desde el cuello hasta las vainas femorales, incluía el riesgo de contaminación/infección por gérmenes entéricos incluidos en el aire inyectado a presión. Ello no ocurrió, pero la evolución infecciosa del paciente fue tórpida, formándose colecciones intraabdominales que requirieron drenajes mediante acceso mínimamente invasivo.

El abordaje quirúrgico puede hacerse de forma mini invasiva o abierta y tendrá como objetivo: cerrar la perforación y derivar el contenido biliar y digestivo $^{15}$.

El tratamiento endoscópico mediante la utilización de clips, endoloops o cola biológica está descrito; postulándose que es menos costoso y con menor estadía hospitalaria que la cirugía, pero aún se requieren estudios con mayor número de casos para recomendarlo ${ }^{14}$.

El manejo conservador está indicado cuando no se logra verificar la lesión, en las perforaciones de tipo II y III y consiste en mantener suspendida la vía oral con eventual nutrición parenteral total y de gravitar el tránsito digestivo mediante la colocación de sonda nasogástrica, antibioticoterapia de amplio espectro, analgesia, tratamiento percutáneo de colecciones, control clínico imagenológico estricto ${ }^{4-6}$. Aunque la mayoría de los casos comunicados en la literatura ${ }^{2,4-6}$ se trataron en forma conservadora, hasta el $20 \%$ de los pacientes en que inicialmente se indica manejo conservador, requiere cirugía en la evolución ${ }^{14}$.

Las indicaciones de cirugía primaria son: perforaciones de tipo I de Stapfer, perforación documen- tada por CPRE con litiasis coledociana no resuelta, extenso enfisema subcutáneo (estas tres presentes en nuestro paciente), peritonitis difusa, extravasación extensa de contraste, perforaciones de tipo II y III que no evolucionan bien con tratamiento conservador $^{14}$.

En perforaciones menores, la sutura debe realizarse de forma transversal, en una o dos capas para prevenir la estenosis asociando epiploplastía. El drenaje enfrentado a la reparación es imprescindible para pesquisar fugas. La aposición serosa y la duodenostomía se reservan para perforaciones de mayor tamaño o más evolucionadas.

La derivación biliar se realiza mediante colecistectomía, colangiografía transcística y degravitación de la vía biliar principal con drenaje transcístico o de Kehr. En nuestro caso se instiló azul de metileno a través de una sonda transcística, que permitió evidenciar la localización y la entidad de la lesión.

La derivación del contenido digestivo mediante técnicas más complejas como la exclusión o diverticulización duodenal se indican en pacientes estables, con perforaciones evolucionadas o ante el fracaso de un tratamiento quirúrgico previo en nuestro caso solamente se emplazó una sonda nasogástrica para degravitación intestinal.

\section{Conclusiones}

La perforación duodenal es una complicación grave e infrecuente de la CPRE con riesgo de complicaciones infecciosas, incluso cuando es diagnosticada y operada precozmente.

El mecanismo de producción por barotrauma, se postula frente a una extensa distribución del aire en los espacios comunicados a través del retroperitoneo y se vincula a la inyección intraduodenal sostenida de aire y/o a la aplicación de alta presión, que puede asociarse o no con una perforación visceral evidente.

La experiencia del endoscopista y la revisión minuciosa del duodeno son fundamentales, ante la sospecha deben agotarse los estudios intraoperatorios e imagenológicos para visualizar fugas de contraste que permitan identificar la perforación.

El tratamiento conservador o la cirugía deben decidirse según el tipo de perforación y la condición clínica del paciente, sabiendo que en ambos casos se requiere control clínico-imagenológico estricto. El cierre de la perforación duodenal, mediante sutura y epiploplastía es un procedimiento seguro y rápido. Técnicas más complejas de exclusión duodenal, deben considerarse frente al fracaso de tratamientos previos. 


\section{Responsabilidades éticas}

Protección de personas y animales. Los autores declaran que para esta investigación no se han realizado experimentos en seres humanos ni en animales.
Confidencialidad de los datos. Los autores declaran que en este artículo no aparecen datos de pacientes.

Conflictos de interés: no hay.

\section{Bibliografía}

1. Cirocchi R, Kelly MD, Griffiths EA, Tabola R, Sartelli M, Carlini L, et al. A systematic review of the management and outcome of ERCP related duodenal perforations using a standardized classification system. The Surgeon xxx 2017;1:e9. Available online at www. sciencedirect.com

2. Schepers N, Van Buuren H. Pneumothorax Following ERCP: Report of Four Cases and Review of the Literature Dig Dis Sci. 2012;57:1990-5. DOI: 10.1007/s10620-012-2150-3.

3. Yilmaz B, Charles Roach E, Koklu S, Aydin O, Unlu O, Alper Kilic Y. Air leak syndrome after endoscopic retrograde cholangiopancreatography: A rare and fatal complication. World J Gastroenterol. 2015;21:4770-2. ISSN 1007-9327 (print) ISSN 2219-2840 (online).

4. Alexiou K, Sakellaridis T, Sikalias N, Karanikas I, Economou N, Antsaklis G. Subcutaneous emphysema, pneumomediastinum and pneumoperitoneum after unsuccessful ERCP: a case report. Cases Journal 2009;2:120 doi: 10.1186/1757-1626-2-120.

5. Doerr R, Kulaylat N, Booth MC, Corasanti J. Barotrauma complicating duodenal perforation during ERCP. Surg
Endosc. 1996;10:349-51.

6. Papamichaila M, Nikolaidisb N, Anastasiouc E, Sidirokastritisc G, Prigourisc P. Massive Subcutaneus Emphysema following Endoscopic Retrograde Cholangiopancreatography with Sphincterotomy. Case Rep Gastroenterol. 2010;4:399-403. DOI: $10.1159 / 000320669$

7. Motomura Y, Akahoshi K, Gilbo G, Kanayama K, Fukuda S, Hamada S, et al. Inmediate detection of endoscopic retrograde chlangiopncreatograhy related periampulary perforations:endoscopy or fluoroscopy. World J Gastroenterol. 2014;20:15797-804. doi: 10.3748/wjg. v20.i42.15797.

8. Paspatin GA, Damouceau JM, Barthat M, Meisner S, Repici A, Saunders BP, et al. Diagnosis and management of iatrogenic endoscopic perforation. European Society of Gastroenterology Endoscopy (ESGE). Position Statement. Endoscopy 2014;46:693-711.

9. William EJ, Taylor S, Fairclought P, Hamlyn A, Logan RF, Martin D, et al. Risk factors for complication following ERCP: Result of a large-scale prospective multicenter study. Endoscopy 2007;39:793-801.

10. Zárate A, Reyes R, Mariscal C. Factores de riesgo predictores para complicaciones post colangiopancreatografía endoscópica retrógrada Cir Endosc. 2016;17:21-3.

11. Kiswani R, Quimseya B, Dweyer L, Wani S. Association betwen endoscopist and centres endoscopic retrograde cholangiography volume with procedures succes and adverse outcomes: A systematic review and meta-analisys. Clin Gastroenterol Hepatol. 2017;15:1866-75.

12. Stapfer M, Selby RR, Stan SC, Kalikhuda N, Parekh D, Jobbour N, et al. Management of duodenal perforation after endoscopic retrograde cholangiography and sphinterectomy. Ann Surgery 2000;232:191-8.

13. Ferrara F, Lugiano C, Bili P, Jovine $\mathrm{E}$, Cinquantini F, D'Imperio N. Phneumotorax, phneumomediastino,pne umoperitoneum, pneumoretroperitoneum and subcutaneus enphisema after ERCP. Gastroint Endosc. 2009;69:1398-401.

14. Morgan KA, Fontenot BB, Rudy JM, Mickey S. Endoscopic retrograde cholangiopancreateography gut perforations: when to wait! When to operated. Am Surg. 2009;75:477-83.

15. Almada M, Martínez J, Muniz N, Bonilla F, Misa R. Perforación duodenal post colangiopancreatografía endoscópica retrógrada. Manejo mini invasivo, reporte de un caso. Cir Urug. 2019;3:10-6. 